\title{
Magnetic Resonance Compatibility of a Transponder Aimed for Radiotherapy Positioning - A Phantom Study
}

\author{
JOHAN WIKSTROM $^{1}$, ULF ISACSSON ${ }^{2}$, BENGT JOHANSSON ${ }^{3}$ and BO LENNERNÄS ${ }^{3}$ \\ ${ }^{1}$ Department of Surgical Sciences, Section of Radiology, Uppsala University, Uppsala, Sweden; \\ ${ }^{2}$ Department of Immunology, Genetics and Pathology, \\ Section of Medical Radiation Sci-ence, Uppsala University, Uppsala, Sweden; \\ ${ }^{3}$ Department of Oncology, Örebro University Hospital, Örebro, Sweden
}

\begin{abstract}
Background/Aim: Electromagnetic Positioning Systems (EMP) is a new position-ing technique in fourdimensional radiotherapy. Patients with implanted transponders may be referred for magnetic resonance imaging (MRI) making it important to establish the MR safety. Materials and Methods: Oranges were prepared with transponders and imaged on a $3 T M R$ scanner with different sequences. Computed tomography (CT) was performed as comparison. MR artifacts were assessed. An estimation of the maximum transponder de-flection force and heating was made. Results: The mean measured displacement of transponders was $0.1 \mathrm{~mm}$ (range $=0.03-0.3 \mathrm{~mm}$ ). Artifacts were observed adjacent to transponders using all sequences. The deflection force on the transponder in the gantry was less than $38 \mathrm{mN}$. No heating was observed. Conclusion: The absence of any substantial movement, the weak measured deflection force and absence of observed heating speaks for the safe use of MR imaging with transponder 3T. Local artefacts makes evaluation impossible adjacent to transponders.
\end{abstract}

Prostate cancer (PC) is one of the most common types of cancer in western countries (1). Modern research and development in surgery and radiation treatment techniques have contributed to significantly improved outcomes for patients who are diagnosed with PC. In recent years hypofractionated curative radiotherapy of $\mathrm{PC}$ has been discussed in order to reduce costs, side effects and possibly improve local control by dose-escalation (2).

Accurate positioning of a patient during a course of external radiotherapy, espe-cially during hypofractionation, is important

Correspondence to: Bo Lennernäs, Department of Oncology, University Hospital Örebro, 70185 Örebro Ho, Sweden. E-mail: bo.lennernas@telia.com

Key Words: Prostate cancer, electromagnetic positioning, four dimensional radiotherapy. to avoid insufficient dosage in the target vol-ume or high doses in organs at risk $(3,4)$. A general dilemma is that high precision de-vices, although often well adapted for special radiation treatments tend to be complicated and unsuitable for standard clinical external beam radiotherapy. Furthermore, few sys-tems, if any, have had the possibility to positionate the target with high precision through-out the whole radiotherapy chain (from diagnosis to the last second of the treatment) with a continuous target position determination.

Electromagnetic Positioning Systems (EMP) technique for 4DRT was first suggested and developed by Lennernäs and Nilsson (5-7) during the 90's and today this is the base of the Raypilot system further developed by Micropos Medical (Gothenburg, Sweden) for clinical use in prostate cancer. This consists of a ferrite core surrounded by a coil. The configuration would be expected to interact with the field of an magnetic reso-nance imaging (MRI) scanner and hence causing both image degradation and the possibility for movements. Since patients with a Raypilot implant might be examined with MRI, either in the follow-up of the prostate cancer or for other indications, the safety and degree of image artefacts caused by the system is important to establish. The purpose of this study was, therefore, to perform an MRI experiment of the Raypilot system in a phantom model in order to assess movements and degree of image degradation.

\section{Materials and Methods}

Three oranges were prepared with one, two and three transponders respectively. The fruits were placed in a water filled plastic box and scanned with the standard MRI protocol used at our institution for evaluation of prostate cancer, including T1-, T2- and diffusion weighted images. T2 turbo spin echo (TSE) sequences were performed in axial, coronal and sagittal planes, T1-weighted TSE and diffusion weighted (DWI) echo-planar imaging (EPI) sequences were performed in the axial plane (details of the sequence parameters are given in Table I). For all obtained sequences, an evaluation was made of the size and form of the artefact associated with the transponder. 


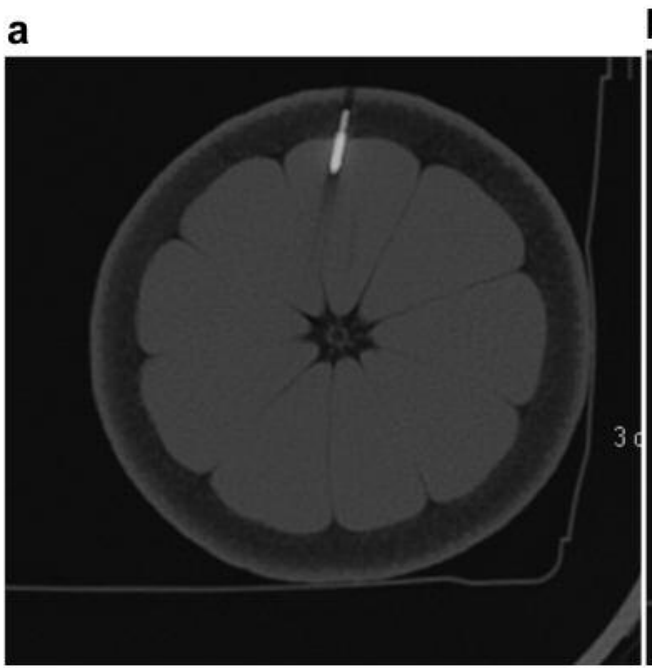

\section{b}

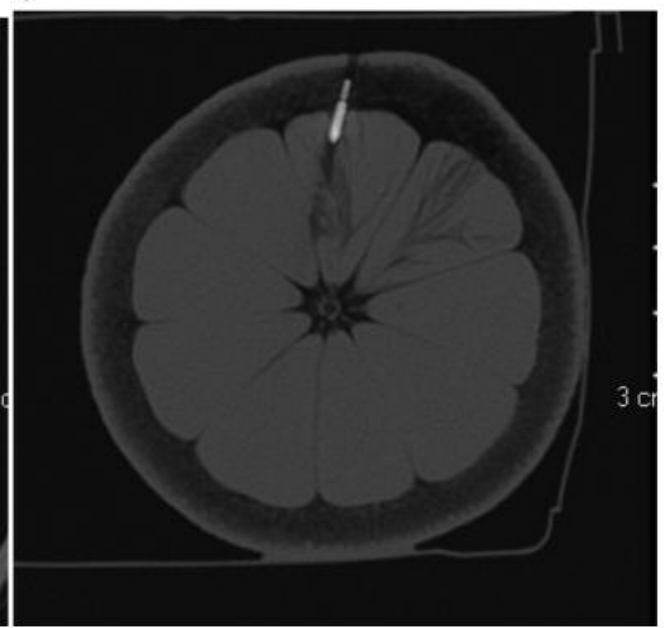

Figure 1. Computed tomographic (CT) images of orange with transponder, before (a) and after $(b)$ MRI.

In order to detect any movement of the transponders associated with the MRI pro-cedure, the fruit box was imaged in a CT scanner before and after the MR investigation. This was done on a 256 slice dual source CT scanner (Siemens Flash, Siemens Medical System, Erlangen, Germany) with a slice thickness of $0.5 \mathrm{~mm}$. Distances between transponders and clearly identifiable reference structures in the oranges were measured and compared between the pre- and post-MRI images. The mean absolute value of the differ-ence between pre- and post-MRI investigations was calculated.

For assessment of the maximum deflection force on the transponder (at the entry of the MRI gantry), the deflection angle of the transponder from the vertical orientation with an object of known mass was measured. A deflection angle of less than 45 degrees would here correspond to a deflection force of less than $\mathrm{m}^{*} \mathrm{~g}(\mathrm{~N})$.

For assessment of possible heating of the transponder during MR imaging; we per-formed a routine clinical prostate protocol including T1-, T2-, and diffusion weighted im-ages in different planes with the transponder fixed to a saline bag. Immediately after imag-ing, the surface temperature of the transponder was assessed by digital examination.

\section{Results}

An example of CT images at the same position before and after MRI is given in Figure 1. The mean distance between the transponder and the chosen reference structure was 1.7 $\mathrm{mm}$ (range=0.7-3.4 mm) before MRI and $1.7 \mathrm{~mm}$ (range $=1.1-3.3 \mathrm{~mm}$ ) after MRI. The mean absolute difference in distance between the transponders and the reference structures between pre- and post-MRI CT images was $0.1 \mathrm{~mm}$ (range $=0.03-0.3 \mathrm{~mm}$ ).

Examples of the different MRI sequences are given in Figure 2. Substantial artefac-tual signal loss was observed adjacent to the transponders. Largest artefacts were ob-served
Table I. Sequence parameters.

\begin{tabular}{lccccc}
\hline Sequence & $\begin{array}{c}\text { TR } \\
(\mathrm{ms})\end{array}$ & $\begin{array}{c}\text { TE } \\
(\mathrm{ms})\end{array}$ & $\begin{array}{c}\text { Flip } \\
\text { angle }\left({ }^{\circ}\right)\end{array}$ & $\begin{array}{c}\text { Spatial } \\
\text { re-solution }(\mathrm{mm})\end{array}$ & $\begin{array}{c}\text { b-value } \\
\left(\mathrm{s} / \mathrm{mm}^{2}\right)\end{array}$ \\
\hline T1 TSE & 519 & 8 & 90 & $0.8 \times 0.9 \times 3$ & NA \\
T2 TSE & 3028 & 100 & 90 & $0.8 \times 1.0 \times 3$ & NA \\
DWI & 1848 & 58 & 90 & $2.3 \times 2.3 \times 3$ & 1000 \\
\hline
\end{tabular}

with the DWI sequence. In the oranges with two or three transponders, artefacts totally obscured the internal architecture in the DWI sequence, whereas in the orange with one transponder, parts of the fruit was possible to discriminate. The size and form was dependent on the slice orientation of the sequence, as exemplified in Figures 3 and 4.

With a plastic clip weighing $3.8 \mathrm{mg}$ attached to the transponder; a deflection angle of less than 45 degrees from the vertical orientation was measured; corresponding to a deflection force of less than $38 \mathrm{mN}$. After scanning there was no observed heating of the transponder.

\section{Discussion}

In this study we performed a phantom experiment to evaluate the possibility of performing MRI in the presence of transponders used for spatial localisation during radiotreatment of prostate cancer. Using clinically-relevant sequence parameters at 3T, no clinically-relevant transponder movements were observed. On the other hand, substantial artefacts were observed in the vicinity of the transponders, 
a

b

C

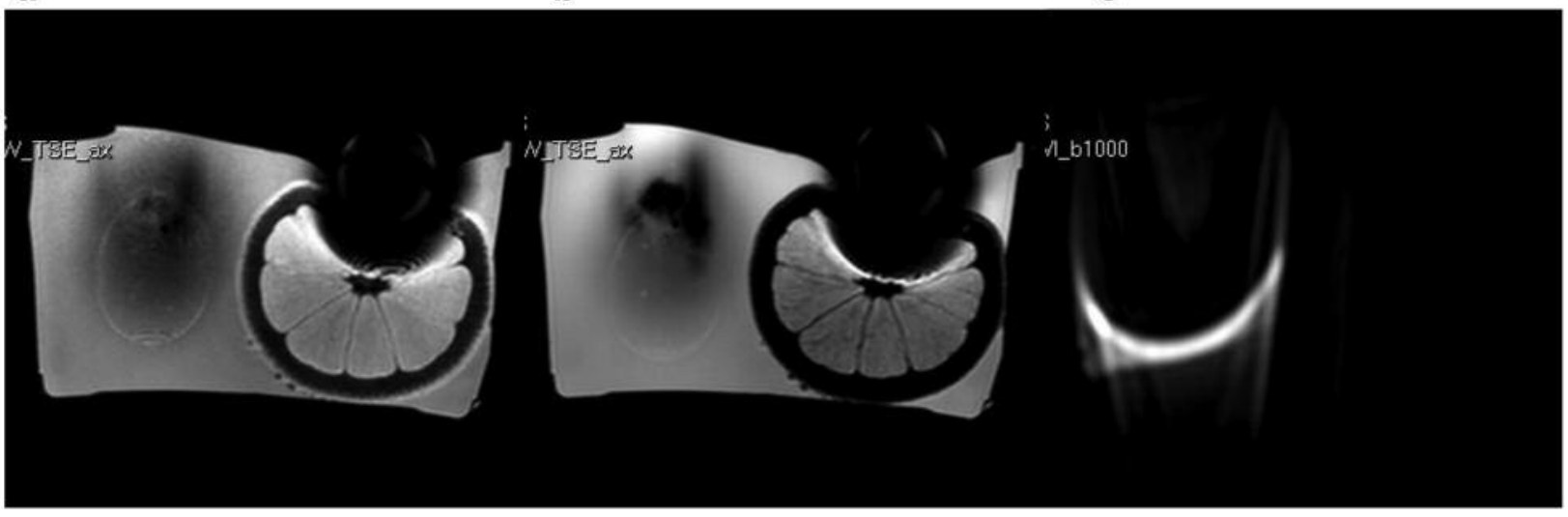

Figure 2. T1- (a), T2- (b) and diffusion weighted (c) images of orange (same as in Figure 1) with inserted transponder.

a

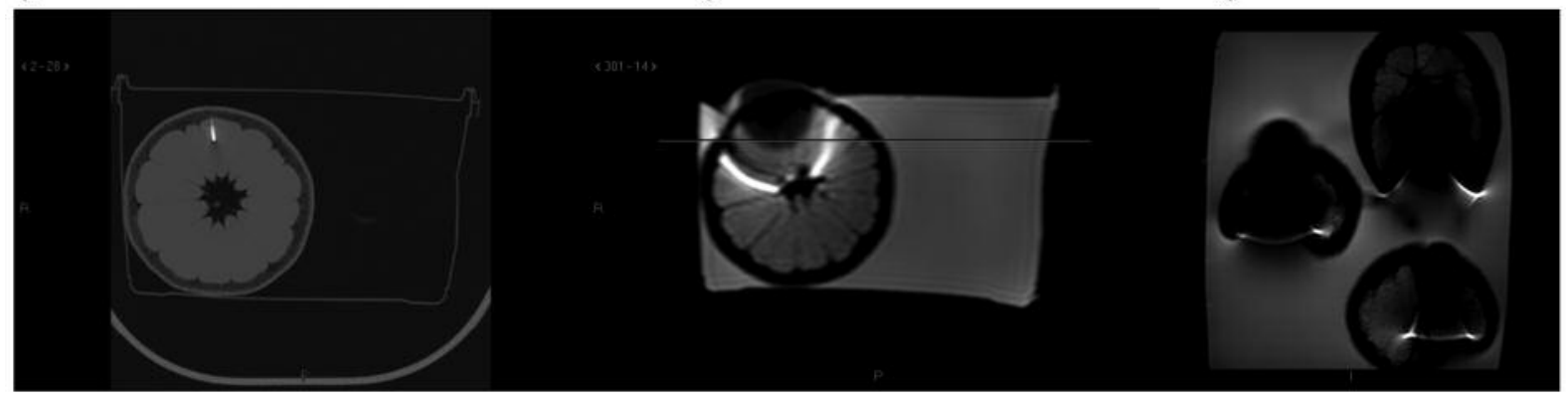

Figure 3. CT image (vertical slice) of transponder (a), with corresponding T2-weighted images in vertical (b) and horizontal (c) orientations, illustrating different shape of artefacts with different slice orientations.

limiting the possibility of MRI for monitoring of therapy efficacy in patients treated for prostate cancer with remaining transponders. We found the ferrite component to be responsible for the artefacts, with no negative effect on image quality when only the coil was present.

In order to assess any movements occurring due to interaction between the trans-ponders and the magnetic and radiofrequency fields, a CT scan was performed of the phantom both before and after the MRI investigation. Comparisons of distances between the transponders and clearly identifiable structures in the oranges before and after MRI showed only minimal differences, likely to be under the detection limit of true movements with the herein used experiment set-up. The absence of any substantial transponder movements and the weak measured deflection force support the safe use of MRI up to 3T in patients with these implants.

In order to assess the possibility of evaluating MRI studies performed of regions adjacent to transponders, we used sequences from our routine $3 \mathrm{~T}$ protocol for prostate cancer.
The studied transponder is made up of a ferrite core with metal wiring. This con-figuration would be expected to cause magnetic susceptibility effects, causing image arte-facts. Major artefacts were also observed adjacent to the transponders using all MRI se-quences. Artefacts were largest using the DWI sequence, which is as expected, this being an echo-planar imaging sequence that is very sensitive to magnetic susceptibility effects. In recent years non-echo planar DWI imaging sequences are being used in some areas. These are slower than EPI-based sequences, but less prone to susceptibility related arte-facts, and might be a better alternative for imaging in the presence of transponders.

With the TSE sequences, smaller, but still substantial artefacts were seen. There were differences in size and shape of the artefacts between sequences with different slice orientation. This is most likely an effect of both transponder orientation relative to the magnetic field and of differences in phase encoding direction. In the presence of trans-ponders both these parameters should be taken into careful 


\section{a}

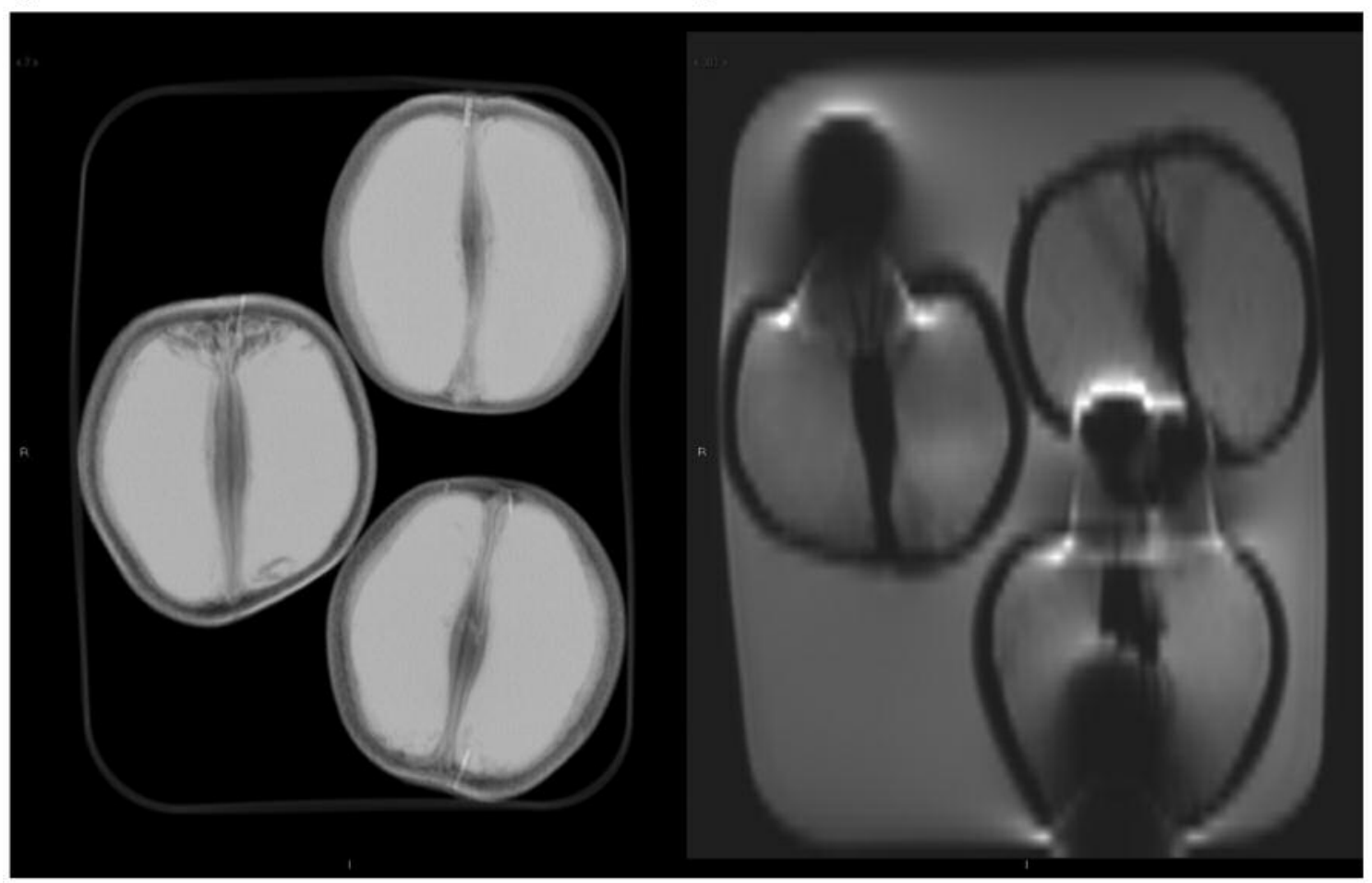

Figure 4. Horizontal multiplanar reformatations of box with oranges at CT (a) and T2-weighted MR (b). Top right orange is prepared with two coils (only one seen in image). Left orange is prepared with ferrites at different orientations (only one seen in image), and bottom right orange is prepared with three ferrites of different lengths. Note absence of artefacts in orange with only coil part of transponder. Size of artefacts is somewhat smaller with decreasing size of ferrite. (Note that bottom right orange has rotated between scan, with smallest artefact corresponding to smallest ferrite).

consideration in order to maximize visualization of the relevant anatomical structures. Our findings regarding both absence of relevant transponder displacement and regarding presence of substantial arte-facts are in good agreement with a previous study on a different real-time tracking device (8).

In conclusion, our results support the safe use of the system at magnetic field strengths of up to 3T. Using standard clinical sequences, there were however major image degradation in the vicinity of ferrites, limiting the possibility of examining the prostate with MRI in the presence of ferrite containing transponders.

\section{References}

1 Adami HO: The prostate cancer pseudo-epidemic. Acta Oncol 49(3): 298-304, 2010.

2 Budiharto T, Haustermans $\mathrm{K}$ and Kovacs G: External beam radiotherapy for pros-tate cancer. J Endourol 24(5): 781-789, 2010.

3 Lennernas B: Patient positioning using magnetic implants and magnetic field sen-sors. Acta Oncol 36(7): 779-781, 1997.

4 Lennernas B, Castellanos E, Nilsson S and Levitt S: Four and five dimensional ra-diotherapy with reference to prostate cancer-definitions, state of the art and further directions--an overview. Acta Oncol 50(Suppl 1): 104-110, 2011.
5 Lennernas B, Edgren M and Nilsson S: Patient positioning using artificial intelli-gence neural networks, trained magnetic field sensors and magnetic implants. Acta Oncol 38(8): 1109-1112, 1999.

6 Lennernas B. and Nilsson S: A new patient positioning system using magnetic im-plants and magnetic field sensors. Radiother Oncol 37(3): 249-250, 1995.

7 Kupelian P, Willoughby T, Mahadevan A, Djemil T, Weinstein G, Jani S, Enke C, Solberg T, Flores N, Liu D, Beyer D and Levine L: Multi-institutional clinical experi-ence with the Calypso System in localization and continuous, real-time monitoring of the prostate gland during external radiotherapy. Int J Radiat Oncol Biol Phys 67(4): 1088-1098, 2007.

8 Zhu X, Bourland JD, Yuan Y, Zhuang T, O'Daniel J, Thongphiew D, Wu QJ, Das SK, Yoo S and Yin FF: Tradeoffs of integrating real-time tracking into IGRT for pros-tate cancer treatment. Phys Med Biol 54(17): N393-401, 2009.

Received April 6, 2017

Revised June 16, 2017

Accepted July 12, 2017 